\title{
Hematopoietic lineage cell-specific protein 1 immunoreactivity indicates an increased risk of poor overall survival in patients with ovarian carcinoma
}

\author{
WENTING LIU ${ }^{1,2}$, HIROAKI KAJIYAMA ${ }^{2}$, KIYOSUMI SHIBATA ${ }^{2}$, \\ YOSHIHIRO KOYA ${ }^{1,2}$, TAKESHI SENGA ${ }^{3}$ and FUMITAKA KIKKAWA ${ }^{2}$ \\ ${ }^{1}$ Bell Research Center for Reproductive Health and Cancer; ${ }^{2}$ Department of Obstetrics and Gynecology; \\ ${ }^{3}$ Division of Cancer Biology, Nagoya University Graduate School of Medicine, Nagoya, Aichi 4668550, Japan
}

Received December 30, 2016; Accepted October 13, 2017

DOI: $10.3892 / \mathrm{ol} .2018 .8493$

\begin{abstract}
Hematopoietic lineage cell-specific protein 1 (HS1) is a $75-\mathrm{kDa}$ intracellular protein that is expressed primarily in hematopoietic cells. Several previous studies have demonstrated the association between HS1 expression and a poor prognosis in hematopoietic malignancies; however, in solid tumors, no studies not been reported. The present study examined the distribution and expression of HS1 in human epithelial ovarian carcinoma (EOC) to determine its clinical significance. Paraffin sections were obtained from EOC tissues and immunostained with HS1 antibody, and then the staining intensities were evaluated. Overall survival (OS) was determined using the Kaplan-Meier estimator method, and multivariate analysis was performed using the Cox proportional hazards analysis. In total, 195 patients with EOC (median age, 56 years) were enrolled into the present study. HS1 immunoreactivity was categorized based on expression levels: Low (89/195; 45.6\%) and high (106/195; 54.4\%). Results demonstrated no association between expression level(s) and any clinicopathological parameter including age, International Federation of Gynecology and Obstetrics (FIGO) staging, type of chemotherapy or type of surgery received. The 5-year OS rates of patients who demonstrated low $(n=89)$ and high $(n=106)$ HS1 expression were 90.4 and $66.7 \%$, respectively. The OS times for patients with high HS1 expression were significantly shorter compared with those for patients exhibiting low HS1 expression $(\mathrm{P}=0.0065)$. Results obtained from the multivariate analysis demonstrated that the FIGO stage and the amount of HS1 expressed were significant independent prognostic markers for poorer OS (hazard ratio, 3.539; $95 \%$ confidence
\end{abstract}

Correspondence to: Professor Hiroaki Kajiyama, Department of Obstetrics and Gynecology, Nagoya University Graduate School of Medicine, 65 Tsuruma-cho, Showa, Nagoya, Aichi 4668550, Japan E-mail: kajiyama@med.nagoya-u.ac.jp

Key words: epithelial ovarian cancer, hematopoietic lineage cell-specific protein 1 , immunohistochemistry, overall survival interval, 1.221-12.811; $\mathrm{P}=0.0187)$. High HS1 expression levels may serve as a useful biomarker in patients with EOC who are likely to exhibit an unfavorable clinical outcome.

\section{Introduction}

Epithelial ovarian cancer (EOC) is the most lethal type of gynecological malignancy. In 2015, 21,290 patients were diagnosed with EOC, and 14,180 succumbed to the disease in the USA (1). Despite the majority of patients with EOC being asymptomatic in the early stages of the disease, this malignancy is frequently associated with multiple intraperitoneal disseminations and distant metastases (2-4). Due to advances in therapeutic strategies, including the development of maximal cytoreductive surgery and several types of effective chemotherapy, it is possible to achieve clinical remission in patients with advanced epithelial ovarian cancer (5). Furthermore, the short-term oncological outcome of women with EOC appears to be more favorable when compared with that of men; however, the majority of clinically complete responders experience disease recurrence $(2,3)$.

Hematopoietic lineage cell-specific protein 1 (HS1) is a 75-kDa multi-domain protein that is primarily expressed within the hematopoietic lineage (6-8). HS1 is frequently regarded as an $\mathrm{F}$-actin binding protein that activates the actin-related protein-2/3 (Arp2/3) complex involved in cytoskeleton rearrangement (6). HS1 is also a signal transducer that acts via the non-receptor-type tyrosine kinases of the Src family causing tyrosine residue phosphorylation (9). Previous studies have demonstrated that HS1 is involved in cell growth, proliferation, adhesion and migration, such as B cells (10), dendritic cells (11), natural killer cells (12), leukemic CLL cells (13) and leukemic B cells (8) that are involved in intracellular signaling. Furthermore, studies have identified that HS1 is expressed on platelets and natural killer cells, as well as in numerous types of hematological malignancies, including acute/chronic leukemia and B-cell chronic lymphocytic leukemia (CLL) (12,14-16).

As a hematopoietic homolog of cortactin, HS1 and cortactin demonstrate a marked similarity in their amino acid sequence and structure $(17,18)$. HS1 and cortactin share an N-terminal acidic domain containing the Arp2/3 complex 
and demonstrate F-actin binding through a 37-amino-acid repeat domain containing three and a half repeats of cortactin; the C-terminal of HS1 is highly homologous (86\%) at the Src homology 3 domain $(6,17,19)$. Despite similarities in homology, HS1 and cortactin exert different biological functions. Cortactin is widely expressed in all types of cell and/or tissue with the exception of hematopoietic cells, and is associated with a poorer clinical prognosis in patients with different types of cancer including hepatocellular carcinoma, laryngeal cancer, ovarian cancer, colon cancer and non-small cell lung cancer (7,20-25). However, to the best of our knowledge, there have been no studies investigating an association between HS1 expression and oncological outcome in solid tumors.

In the present study, the expression levels of HS1, and the potential association between HS1 expression and clinicopathological features in four common types (serous, clear cell, mucinous and endometrioid carcinoma) of EOC were investigated in patients with EOC.

\section{Materials and methods}

Patients and tissue samples. Tissues were obtained from 195 patients who underwent initial surgery at the Nagoya University Hospital (Nagoya, Aichi, Japan) between January 1999 and December 2011. Patients who underwent pre-surgical treatment including radiotherapy and chemotherapy, were excluded from the present study. All patients provided informed consent prior to recruitment. Surgical treatment consisted of total hysterectomy, bilateral salpingo-oophorectomy, omentectomy, and pelvic and para-aortic lymphadenectomy. In the situation where residual tumor remained, systemic lymphadenectomy was omitted. The histological type was assigned according to criteria outlined by the World Health Organization classification (2003) (26). Clinical staging was reviewed based on staging criteria of the International Federation of Gynecology and Obstetrics 1988 (FIGO) (27). All tissue samples were fixed in $10 \%$ neutral buffered formalin for 24 to $48 \mathrm{~h}$ at room temperature, embedded in paraffin and routinely stained with hematoxylin and eosin for histological examination.

Immunohistochemical HS1 staining and evaluation. Formalin-fixed, paraffin-embedded tissue blocks (4- $\mu \mathrm{m}$ thick) were mounted on charged glass slides, de-paraffinized, and rehydrated in a graded series of ethanol. Antigen retrieval was conducted in $10 \mathrm{mM}$ citrate solution ( $\mathrm{pH}$ 6.0) (Wako Pure Chemical Industries, Ltd., Osaka, Japan) at $98^{\circ} \mathrm{C}$ for $15 \mathrm{~min}$. Endogenous peroxidase activity was blocked with $3 \%$ hydrogen peroxide for $5 \mathrm{~min}$ at room temperature. Blocking was performed in 10\% normal goat serum (Dako; Agilent Technologies, Inc., Santa Clara, CA, USA) with $0.025 \%$ Triton $\mathrm{X}-100$ in Tris-buffered saline (TBS-T) for $60 \mathrm{~min}$. Sections were treated with anti-HS1 rabbit monoclonal antibody in blocking buffer at $4^{\circ} \mathrm{C}$ overnight (1:100; cat. no. 3890; Cell Signaling Technology, Inc., Danvers, MA, USA). Following three washes with TBS-T, sections were incubated with biotinylated goat anti-rabbit (1:200; cat. no. PK-4001; Vector Laboratories, Inc., Burlingame, CA, USA) horseradish peroxidase for $1 \mathrm{~h}$ and incubated with avidin-biotin complex reagent (cat. no. PK-4001; Vector Laboratories, Inc.) for $30 \mathrm{~min}$ at room temperature. Peroxidase activities were visualized using a 3,3'-diaminobenzidine peroxidase substrate kit (cat. no SK-4100; Vector Laboratories, Inc.). Slides were counterstained with Mayer's hematoxylin for $5 \mathrm{~min}$ at room temperature (Wako Pure Chemical Industries, Ltd.). Sections were dehydrated, cleared and mounted. Negative controls were run on all sections in blocking buffer without the primary antibody. The intensity of HS1 immunostaining was scored as follows: 0 (negative), 1 (weak), 2 (intermediate) and 3 (strong). The extent of staining was scored as 0 (0-25\%), 1 (26-50\%), $2(51-75 \%)$ or $3(>76 \%)$ according to the percentage of medium and strong staining in relation to the total cancer area. Specimens with a final staining score of 0 or 1 indicated low HS1 expression, whereas a final staining score of 2 or 3 indicated high HS1 expression. The scoring procedure was performed twice by two independent observers (each blinded to the other's scores) who had no knowledge of the patients' clinical parameters and other prognostic factors. The concordance rate was $>95 \%$ between the observers. For the other 5\% disagreement, it was adjusted by observer's consensus.

Statistical analysis. The $\chi^{2}$ test was performed to analyze the association between low and high HS1 expression and patient clinicopathological parameters. Survival curves were generated using the Kaplan-Meier estimator method, which were then compared using the log-rank test. Overall survival (OS) was defined as the time between the date of initial therapy and the final follow-up or the time of patient mortality due to any cause. The prognostic significance of HS1 expression regarding other pathological variables was analyzed using univariate and multivariate Cox proportional hazards regression analysis. $\mathrm{P}<0.05$ was considered to indicate a statistically significant difference.

\section{Results}

Patient characteristics. In total, 195 patients with EOC were enrolled into the present study. The age range of diagnosed patients was between 20 and 82 years (median, 56 years). According to the results presented in Table I, the FIGO stage distribution was as follows: 81 stage I patients $(41.5 \%)$, 17 stage II patients $(8.7 \%), 60$ stage III patients $(30.8 \%)$ and 13 stage IV patients $(6.7 \%)$. Staging could not be determined in 24 patients (12.3\%), due to incomplete records. With regard to the pathological type, the serous histological type was the most frequently identified $(81 / 195 ; 41.5 \%)$, followed by clear cell carcinoma $(61 / 195 ; 31.3 \%)$. A total of $162(83.1 \%)$ patients were administered using Taxane plus platinum chemotherapy (paclitaxel plus cisplatin, paclitaxel plus carboplatin, docetaxel plus cisplatin, docetaxel plus carboplatin), the remaining $33(16.9 \%)$ cases were not treated. In total, $79(40.5 \%)$ patients underwent full staging and/or complete surgery, and 116 (59.5\%) underwent unstaged and/or debulking surgery. Table I summarizes all patient characteristics.

HS1 expression in EOC samples. As presented in Fig. 1, high and low levels of HS1 expression were identified in EOC samples. Results demonstrated that HS1 protein was localized in the cytoplasm and membrane of the all stages tumor cells. 
Table I. Patient characteristics.

\begin{tabular}{lc}
\hline Clinical parameter & $\mathrm{n}(\%)$ \\
\hline FIGO Stage & \\
I & $81(41.5)$ \\
II & $17(8.7)$ \\
III & $60(30.8)$ \\
IV & $13(6.7)$ \\
Unspecified & $24(12.3)$ \\
Histological type & \\
Serous & $81(41.5)$ \\
Clear-cell & $61(31.3)$ \\
Endometrioid & $39(20.0)$ \\
Mucinous & $14(7.2)$ \\
Chemotherapy & \\
Taxane plus platinum & $162(83.1)$ \\
Others & $33(16.9)$ \\
Surgery & \\
Full staging/complete & $79(40.5)$ \\
Non-staging/debulking & $116(59.5)$ \\
HS1 expression & \\
Low & $106(54.4)$ \\
High & \\
\hline
\end{tabular}

FIGO, International Federation of Gynecology and Obstetrics; HS1, hematopoietic lineage cell-specific protein 1 .

According to results present in Table II, the distribution of the HS1 immunostaining intensity was as follows: 34 patients with negative staining (score $0 ; 17.4 \%$ ), 55 patients with weak staining (score $1 ; 28.2 \%$ ), 86 patients with intermediate staining (score $2 ; 44.1 \%$ ) and 20 patients with strong staining (score 3; 10.3\%). Tumors were categorized based upon the staining score. Low HS1 expression indicated a staining score of $0-1(89 / 195 ; 45.6 \%)$ and high HS1 expression indicated a staining score of 2-3 (106/195; 54.4\%). As presented in Table II, high HS1 immunoreactivity was detected in the following histological types: 43 serous $(43 / 81 ; 53.1 \%)$, 41 clear cell (41/61; 67.2\%), 18 endometrioid $(18 / 39 ; 46.2 \%)$ and 4 mucinous $(4 / 14 ; 28.6 \%)$ carcinoma cases. The HS1 immunoreactivity categorized into low vs. high expression was not associated with any of the clinicopathological parameters, including age, FIGO stage and type of chemotherapy or surgery received; however, histological type was significantly associated with HS1 expression $(\mathrm{P}=0.0303)$ (Table III).

Association between HS1 immunoreactivity and oncological outcome of patients with EOC. The association between HS1 expression and OS was investigated. The median follow-up time of the patients was 67.2 months. At the end of the follow-up period, 166/195 (85.1\%) patients remained alive and $29 / 195$ (14.9\%) patients had succumbed to the disease. The 3- and 5-year OS rates of all patients were 80.3 and $75.8 \%$, respectively. The 5-year OS rates of patients with low $(n=89)$ and high $(n=106)$ expression of HS1 were 90.4 and $66.7 \%$,
Table II. Distribution of hematopoietic lineage cell-specific protein 1 immunoreactivity scores for each EOC histological type.

\begin{tabular}{lrccc}
\hline & \multicolumn{4}{c}{ Immunoreactivity score, $\mathrm{n}(\%)$} \\
\cline { 2 - 5 } Type of EOC & 0 & 1 & 2 & 3 \\
\hline Serous & $12(35.3)$ & $26(47.3)$ & $30(34.9)$ & $13(65.0)$ \\
Clear cell & $4(11.8)$ & $16(29.1)$ & $36(41.9)$ & $5(25.0)$ \\
Endometrioid & $12(35.3)$ & $9(16.4)$ & $16(18.6)$ & $2(10.0)$ \\
Mucinous & $6(17.6)$ & $4(7.3)$ & $4(4.7)$ & $0(0.0)$ \\
\hline EOC, epithelial ovarian carcinoma. & \\
\hline
\end{tabular}

Table III. Distribution of several clinicopathological factors according to HS1 expression.

\begin{tabular}{|c|c|c|c|}
\hline \multirow[b]{2}{*}{ Factor } & \multicolumn{3}{|c|}{ HS1 expression, $\mathrm{n}(\%)$} \\
\hline & Low & High & $\mathrm{P}$-value \\
\hline Age, years & & & 0.334 \\
\hline$\leq 55$ & $27(30.3)$ & $24(22.6)$ & \\
\hline$>55$ & $54(60.7)$ & $75(70.8)$ & \\
\hline Unspecified $^{\mathrm{a}}$ & $8(9.0)$ & $7(6.6)$ & \\
\hline FIGO stage & & & 0.705 \\
\hline I & $38(42.7)$ & $43(40.6)$ & \\
\hline II & $8(9.0)$ & $9(8.5)$ & \\
\hline III & $24(27.0)$ & $36(34.0)$ & \\
\hline IV & $8(9.0)$ & $5(4.7)$ & \\
\hline Unspecified $^{\mathrm{a}}$ & $11(12.4)$ & $13(12.3)$ & \\
\hline Histological type & & & 0.0303 \\
\hline Serous & $38(42.7)$ & $43(40.6)$ & \\
\hline Clear cell & $20(22.5)$ & $41(38.7)$ & \\
\hline Endometrioid & $21(23.6)$ & $18(17.0)$ & \\
\hline Mucinous & $10(11.2)$ & $4(3.8)$ & \\
\hline Chemotherapy & & & 0.719 \\
\hline Taxane plus platinum & $73(82.0)$ & $89(84.0)$ & \\
\hline Others & $16(18.0)$ & $17(16.0)$ & \\
\hline Surgery & & & 0.547 \\
\hline Full staging/complete & $34(38.2)$ & $45(42.5)$ & \\
\hline Non-staging/debulking & $55(61.8)$ & $61(57.5)$ & \\
\hline
\end{tabular}

${ }^{a}$ Unspecified as a result of missing data. FIGO, International Federation of Gynecology and Obstetrics; HS1, hematopoietic lineage cell-specific protein 1 .

respectively. The OS time in patients with high HS1 expression was significantly shorter compared with that in patients with low HS1 expression ( $\mathrm{P}=0.0065$; Fig. 2). Additionally, analysis of patients with a serous histological type revealed that significantly poorer OS was demonstrated in patients with high HS1 expression compared with that in patients with low HS1 expression ( $\mathrm{P}=0.0397$; Fig. 3A). Furthermore, analysis 

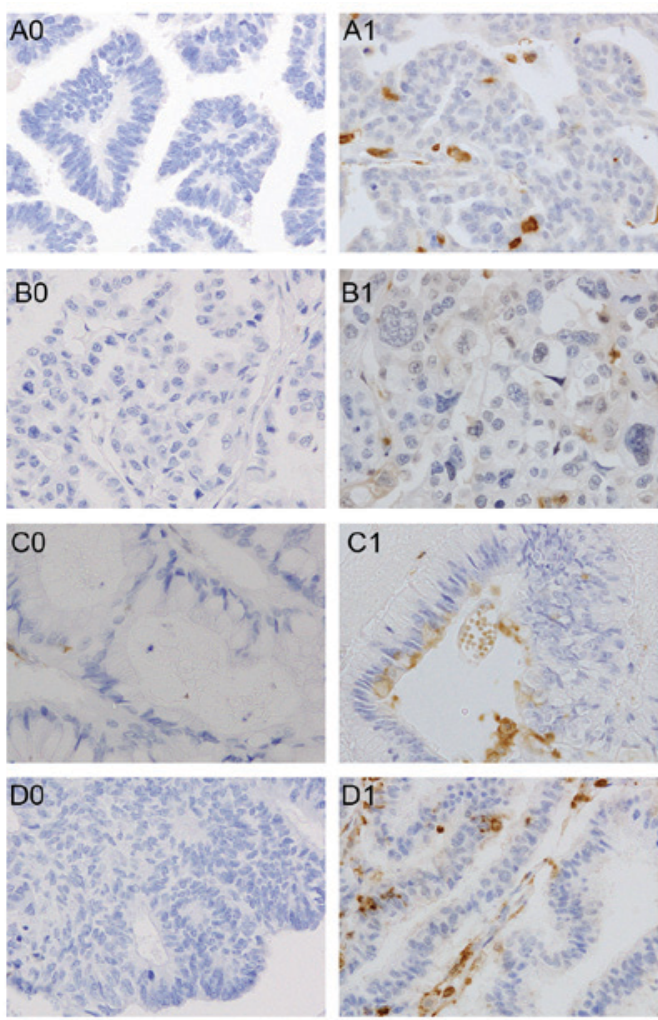
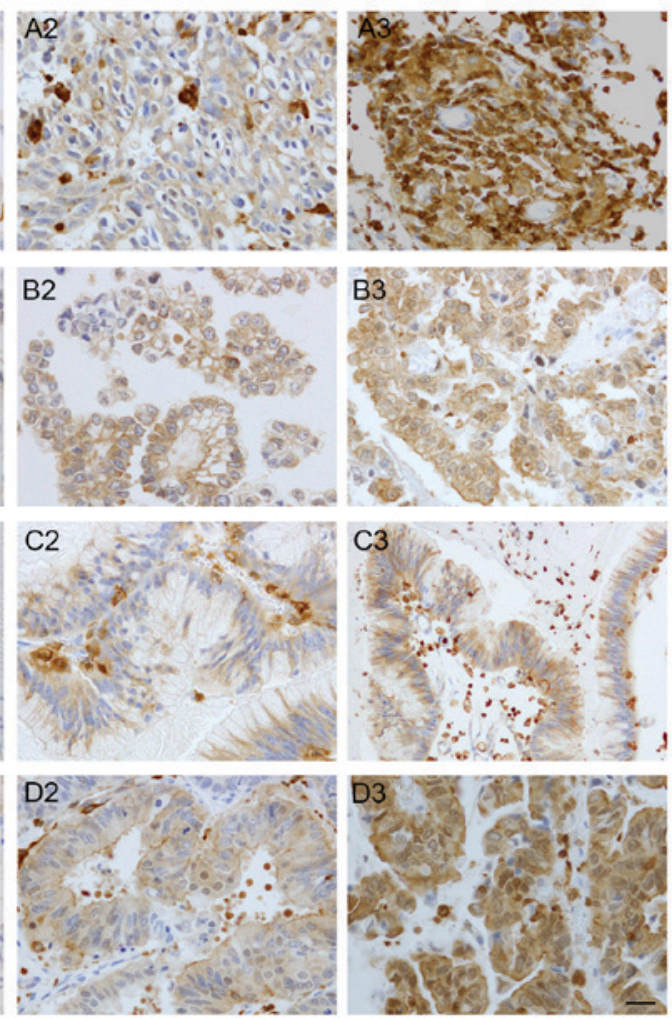

Figure 1. Immunohistochemical staining for hematopoietic lineage cell-specific protein 1 expression in (A) serous carcinoma, (B) clear cell carcinoma, (C) mucinous carcinoma and (D) endometrioid carcinoma. As indicated by numbers 0-3 in the images, immunoreactivity was scored as follows: 0, negative staining; 1, weak staining; 2, intermediate staining; and 3, strong staining. Scale bar, $20 \mu \mathrm{m}$.

of patients with a non-serous histological type demonstrated similar tendencies in the long-term clinical outcome, although the result was not significant $(\mathrm{P}=0.0600$; Fig. 3B).

Furthermore, univariate and multivariate analyses were performed on 170 patients with complete clinical information including age, FIGO stage, histological type, type of chemotherapy and type of surgery; 25 patients with limited clinical information are excluded from this analysis. Results from the univariate analyses demonstrated that the FIGO stage III/IV, serous histological type, non-staging surgical procedure and high HS1 immunoreactivity significantly predicted a poor OS outcome $(\mathrm{P}<0.0001, \mathrm{P}=0.0237, \mathrm{P}=0.0143$ and $\mathrm{P}=0.0320$, respectively). For multivariate analyses, age, FIGO stage, histological type, type of chemotherapy, type of surgery and HS1 immunoreactivity were recruited into the Cox proportional hazards model. Results demonstrated that FIGO staging (hazard ratio, 30.114; 95\% confidence interval, 7.226-205.835; $\mathrm{P}<0.0001$ ) and HS1 expression (hazard ratio, $3.539 ; 95 \%$ confidence interval, 1.221-12.811; $\mathrm{P}=0.0187$ ) were significant prognostic factors for the prediction of a poor OS outcome (Table IV).

\section{Discussion}

To the best of our knowledge, the present study is the first to investigate and identify that HS1 is highly expressed in four representative types (serous, clear cell, mucinous and endometrioid carcinoma) of EOC samples, and that high expression is associated with a poor prognosis. van Rossum et al (19) reported that $\mathrm{HS} 1$ demonstrated a genomic organization

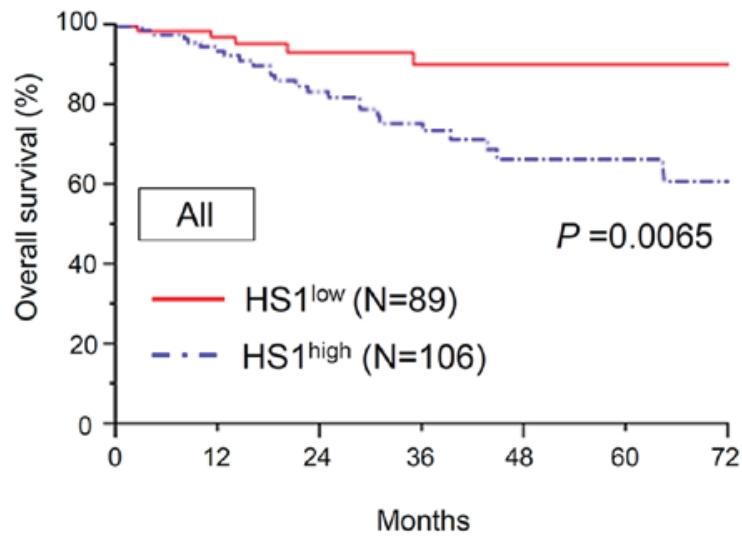

Figure 2. Kaplan-Meier OS curves for patients across all histological types according to HS1 expression (low/high). The continuous line represents the low HS1 immunoexpression group $(n=89)$. The discontinuous line represents the high HS1 immunoexpression group $(n=106)$. Patients with high HS1 expression demonstrated a significantly poorer OS rate compared with those with low HS1 expression $(\mathrm{P}=0.0065)$. HS1, hematopoietic lineage cell-specific protein 1 ; OS, overall survival.

similar to cortactin; however, the comparison between HS1 and cortactin levels in solid tumors is yet to be investigated.

It was previously hypothesized that HS1 and cortactin genes exhibit differing expression patterns; however, in platelets (28) and megakaryocytes (29), cortactin is expressed similarly to HS1. In macrophages (30) and carcinoma cells (31), HS1 and cortactin are accumulated in podosomes. A recent study demonstrated that cortactin is highly expressed and regulates 
Table IV. Univariate and multivariate analysis ${ }^{\mathrm{a}}$ of clinicopathological parameters in association with overall survival ${ }^{\mathrm{b}}$

\begin{tabular}{|c|c|c|c|c|}
\hline \multirow[b]{3}{*}{ Factor } & \multicolumn{4}{|c|}{ Overall survival } \\
\hline & \multicolumn{2}{|c|}{ Univariate } & \multicolumn{2}{|c|}{ Multivariate } \\
\hline & $\mathrm{HR}(95 \% \mathrm{CI})$ & P-value & $\mathrm{HR}(95 \% \mathrm{CI})$ & P-value \\
\hline Age, years & & 0.734 & & 0.887 \\
\hline$\leq 55$ & 1 & & 1 & \\
\hline$>55$ & $0.855(0.340-2.123)$ & & $0.932(0.347-2.485)$ & \\
\hline FIGO stage & & $<0.0001$ & & $<0.0001$ \\
\hline $\mathrm{I} / \mathrm{II}$ & 1 & & 1 & \\
\hline III/IV & $21.611(6.155-136.684)$ & & $30.114(7.226-205.835)$ & \\
\hline Histological type & & 0.0237 & & 0.232 \\
\hline Serous & 1 & & 1 & \\
\hline Non-serous & $0.348(0.138-0.865)$ & & $1.938(0.647-5.734)$ & \\
\hline Chemotherapy & & 0.489 & & 0.182 \\
\hline Taxane plus platinum & 1 & & 1 & \\
\hline Others & $1.587(0.367-4.822)$ & & $2.821(0.576-10.832)$ & \\
\hline Surgery & & 0.0143 & & 0.199 \\
\hline Full staging/complete & 1 & & 1 & \\
\hline Non-staging/debulking & $3.294(1.258-10.207)$ & & $2.038(0.698-6.906)$ & \\
\hline HS1 & & 0.0320 & & 0.0187 \\
\hline Low & 1 & & 1 & \\
\hline High & $3.013(1.093-10.573)$ & & $3.539(1.221-12.811)$ & \\
\hline
\end{tabular}

${ }^{a}$ Cox hazard model; ${ }^{b} 170$ patients with full clinical information including age, FIGO stage, histological type, type of chemotherapy and type of surgery. HR, hazard ratio; CI, confidence interval; FIGO, International Federation of Gynecology and Obstetrics; HS1, hematopoietic lineage cell-specific protein 1 .
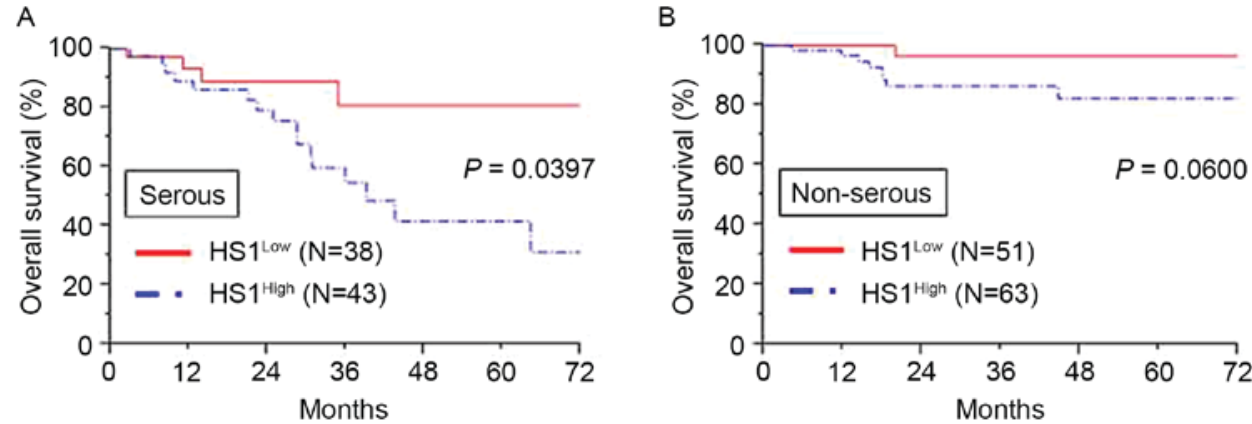

Figure 3. (A) Kaplan-Meier overall survival curves for patients with serous EOC with regard to HS1 immunoexpression. The continuous line represents the low HS1 immunoexpression group ( $\mathrm{n}=38$ ), whereas the discontinuous line represents the high HS1 immunoexpression group ( $\mathrm{n}=43$ ). Patients with high HS1 expression demonstrated a significantly poorer overall survival rate compared with those with low HS1 expression ( $\mathrm{P}=0.0397)$. (B) Kaplan-Meier overall survival curves for patients with non-serous EOC with regard to HS1 immunoexpression. The continuous line represents the low HS1 immunoexpression group (n=51), whereas the discontinuous line represents the high HS1 immunoexpression group (n=63). Overall, patients with high HS1 expression demonstrated a poorer overall survival compared with those with low HS1 expression, although this difference was not significant $(\mathrm{P}=0.0600)$. EOC, epithelial ovarian carcinoma; HS1, hematopoietic lineage cell-specific protein 1.

splicing in patients with B-cell CLL, and is associated with a poor prognosis (32). These contradictory findings suggest that the HS1 gene may possess multiple functions due to being present in different pathological lineages.

Results from the present study demonstrated that HS1 was not expressed in normal ovarian tissue, and the positive expression rate in melanoma was high, with a staining score of 3 (data not shown). Xu et al (33) demonstrated that the aberrant expression of cortactin is associated with melanocytic tumor progression. In the present study HS1 expression was primarily identified in the cytoplasm of serous, clear cell, endometrioid and mucinous carcinoma cells. Additionally, results obtained from the Kaplan-Meier survival analysis indicated that high HS1 expression was significantly associated with a 
decreased survival rate compared with low HS1 expression. In total, $\sim 80 \%$ of ovarian carcinoma samples were of the serous type, and results demonstrated that high HS1 expression was associated with a significant decrease in OS rate compared with low HS1 expression (34). Furthermore, univariate analysis was performed in order to assess associations between prognosis and several clinical characteristics, including the FIGO stage, histological type, type of chemotherapy, type of surgery and HS1 expression level status. Results demonstrated that the advanced stage (stages III and IV), serous histological type, non-staging surgery and high expression of HS1 were significant prognostic markers for poor OS for patients with EOC. Multivariate analysis performed on these parameters using the Cox proportional hazards model revealed that high HS1 expression, EOC tumor type and the FIGO stage were independent prognostic factors for EOC. We hypothesize that HS1 functions similarly in serous and non-serous EOC; however, analysis of the association between OS and non-serous EOC could not be performed due to unbalanced stage distribution (in clear cell carcinoma, there were 47 stage I/II specimens (31 high vs. 16 low HS1 expression) and 13 stage III/IV specimens (10 high vs. 3 low HS1 expression). The unbalanced stage distribution may result in type II error (also known as a 'false negative' finding). We hypothesize that if a larger number of non-serous carcinoma specimens were reanalyzed, HS1 expression may be significantly associated with a poor prognosis.

A previous study demonstrated that HS1 was associated with the remodeling of the actin cytoskeleton (17). Scielzo et al (16) demonstrated that HS1 served important functions in cell migration, bone marrow infiltration and chemotherapy resistance, and therefore was associated with a poor prognosis. Additionally, it was identified that patients with B-cell CLL who exhibited the worst prognosis exhibited highly phosphorylated HS1 protein that was able to infiltrate the bone marrow (8). In a previous animal model, silencing of HS1 expression exerted abnormal cell adhesion and reduced cell migration in immunodeficient mice (8). HS1-deficient mice also demonstrated antigen-receptor-induced apoptosis and a proliferative response of splenic B and T cells (35). Despite no direct evidence of HS1 expression in other types of solid carcinoma, several biomarkers associated with F-actin-bundling protein and anti-apoptosis, including cortactin, fascin and survivin, were expressed and associated with a poor prognosis in patients with EOC $(23,36)$. A previous study demonstrated that fascin is upregulated in numerous types of human carcinoma, and is associated with tumor aggressiveness and poor OS (37). Additionally, previous studies reported that cortactin and HS1 are actin-binding molecules involved in adhesion and cellular migration via actin skeleton remodeling in the majority of cell types $(7,17,19,20,38)$. Cortactin overexpression is associated with invasiveness of various solid tumor cells and a poor prognosis, such as human hepatocellular carcinoma (21), laryngeal carcinomas (22), ovarian cancer (23), colon cancer (24), non-small cell lung cancer (25) and hematopoietic lineages such as in platelets (28), megakaryocytes (29) and B-cell CLL (32). As HS1 is a homolog of cortactin, we hypothesized that HS1 may exhibit a similar role to cortactin with regard to its biological properties and mechanisms in EOC $(17,19)$. Peritoneal metastasis is the most frequent clinical presentation demonstrated in EOC and is composed of multiple stages including release from the original ovarian neoplasm, attachment to the mesothelium and subsequent migration/invasion into the subperitoneal tissue. Future studies are required to investigate the underlying molecular mechanisms of HS1 in ovarian cancer metastasis.

In summary, the results of the present study demonstrate that HS1 is expressed in four common types of EOC and is associated with the OS of patients with EOC. Furthermore, to the best of our knowledge, this is the first report investigating the clinical significance of HS1 in EOC. The present study demonstrates that the immunoreactivity of HS1 is an independent prognostic marker for patients with EOC. In order to assess the role of the HS1 gene further, early clinical detection and novel therapeutic approaches are required in vivo and in vitro.

\section{Acknowledgements}

The authors would like to thank the laboratory team from the Department of Obstetrics and Gynecology, Nagoya University Graduate School of Medicine, for providing tissue samples and technical support.

\section{References}

1. Siegel RL, Miller KD and Jemal A: Cancer statistics, 2015. CA Cancer J Clin 65: 5-29, 2015.

2. Kajiyama H, Shibata K, Mizuno M, Umezu T, Suzuki S, Yamamoto E, Fujiwara S, Kawai M, Nagasaka T and Kikkawa F: Long-term clinical outcome of patients with recurrent epithelial ovarian carcinoma: Is it the same for each histological type? Int J Gynecol Cancer 22: 394-399, 2012.

3. Kikkawa F, Nawa A, Ino K, Shibata K, Kajiyama $H$ and Nomura S: Advances in treatment of epithelial ovarian cancer. Nagoya J Med Sci 68: 19-26, 2006.

4. Yoshikawa N, Kajiyama H, Mizuno M, Shibata K, Kawai M, Nagasaka T and Kikkawa F: Clinicopathologic features of epithelial ovarian carcinoma in younger vs. older patients: Analysis in Japanese women. J Gynecol Oncol 25: 118-123, 2014.

5. Eisenkop SM, Friedman RL and Wang HJ: Complete cytoreductive surgery is feasible and maximizes survival in patients with advanced epithelial ovarian cancer: A prospective study. Gynecol Oncol 69: 103-108, 1998.

6. Kitamura D, Kaneko H, Miyagoe Y, Ariyasu T and Watanabe T: Isolation and characterization of a novel human gene expressed specifically in the cells of hematopoietic lineage. Nucleic Acids Res 17: 9367-9379, 1989.

7. Kitamura D, Kaneko H, Taniuchi I, Akagi K, Yamamura K and Watanabe T: Molecular cloning and characterization of mouse HS1. Biochem Biophys Res Commun 208: 1137-1146, 1995.

8. Scielzo C, Bertilaccio MT, Simonetti G, Dagklis A, Ten Hacken E, Fazi C, Muzio M, Caiolfa V, Kitamura D, Restuccia U, et al: HS1 has a central role in the trafficking and homing of leukemic B cells. Blood 116: 3537-3546, 2010.

9. Yamamoto T, Yamanashi Y and Toyoshima K: Association of Src-family kinase Lyn with B-cell antigen receptor. Immunol Rev 132: 187-206, 1993.

10. Yamanashi Y, Okada M, Semba T, Yamori T, Umemori H, Tsunasawa S, Toyoshima K, Kitamura D, Watanabe T and Yamamoto T: Identification of HS1 protein as a major substrate of protein-tyrosine kinase(s) upon B-cell antigen receptor-mediated signaling. Proc Natl Acad Sci USA 90: 3631-3635, 1993

11. Dehring DA, Clarke F, Ricart BG, Huang Y, Gomez TS, Williamson EK, Hammer DA, Billadeau DD, Argon Y and Burkhardt JK: Hematopoietic lineage cell-specific protein 1 functions in concert with the Wiskott-Aldrich syndrome protein to promote podosome array organization and chemotaxis in dendritic cells. J Immunol 186: 4805-4818, 2011. 
12. Butler B, Kastendieck DH and Cooper JA: Differently phosphorylated forms of the cortactin homolog HS1 mediate distinct functions in natural killer cells. Nat Immunol 9: 887-897, 2008.

13. Caligaris-Cappio F, Bergui L, Tesio L, Corbascio G, Tousco F and Marchisio PC: Cytoskeleton organization is aberrantly rearranged in the cells of $\mathrm{B}$ chronic lymphocytic leukemia and hairy cell leukemia. Blood 67: 233-239, 1986.

14. Thomas SG, Calaminus SD, Auger JM, Watson SP and Machesky LM: Studies on the actin-binding protein HS1 in platelets. BMC Cell Biol 8: 46, 2007.

15. Hussein K, von Neuhoff N, Büsche G, Buhr T, Kreipe H and Bock O: Opposite expression pattern of Src kinase Lyn in acute and chronic haematological malignancies. Ann Hematol 88: 1059-1067, 2009.

16. Scielzo C, Ghia P, Conti A, Bachi A, Guida G, Geuna M, Alessio $\mathrm{M}$ and Caligaris-Cappio F: HS1 protein is differentially expressed in chronic lymphocytic leukemia patient subsets with good or poor prognoses. J Clin Invest 115: 1644-1650, 2005.

17. Hao JJ, Zhu J, Zhou K, Smith N and Zhan X: The coiled-coil domain is required for HS1 to bind to F-actin and activate Arp2/3 complex. J Biol Chem 280: 37988-37994, 2005.

18. He H: HS1 and EMS1. Gan To Kagaku Ryoho 24: 1448-1453, 1997 (In Japanese).

19. van Rossum AG, Schuuring-Scholtes E, van Buuren-van Seggelen V, Kluin PM and Schuuring E: Comparative genome analysis of cortactin and HS1: The significance of the F-actin binding repeat domain. BMC Genomics 6: 15, 2005.

20. Schuuring E, van Damme H, Schuuring-Scholtes E, Verhoeven E, Michalides R, Geelen E, de Boer C, Brok H, van Buuren V and Kluin P: Characterization of the EMS1 gene and its product, human Cortactin. Cell Adhes Commun 6: 185-209, 1998.

21. Zhao G, Huang ZM, Kong YL, Wen DQ, Li Y, Ren L and Zhang HY: Cortactin is a sensitive biomarker relative to the poor prognosis of human hepatocellular carcinoma. World J Surg Oncol 11: 74, 2013.

22. Ambrosio EP, Rosa FE, Domingues MA, Villacis RA, Coudry Rde A, Tagliarini JV, Soares FA, Kowalski LP and Rogatto SR Cortactin is associated with perineural invasion in the deep invasive front area of laryngeal carcinomas. Hum Pathol 42: 1221-1229, 2011

23. Lin CK, Chao TK, Yu CP, Yu MH and Jin JS: The expression of six biomarkers in the four most common ovarian cancers: Correlation with clinicopathological parameters. APMIS 117: $162-175,2009$

24. Ni QF, Yu JW, Qian F, Sun NZ, Xiao JJ and Zhu JW: Cortactin promotes colon cancer progression by regulating ERK pathway. Int J Oncol 47: 1034-1042, 2015.

25. Noh SJ, Baek HA, Park HS, Jang KY, Moon WS, Kang MJ, Lee DG, Kim MH, Lee JH and Chung MJ: Expression of SIRT1 and cortactin is associated with progression of non-small cell lung cancer. Pathol Res Pract 209: 365-370, 2013.

26. Tavassoli FA, Devilee, Peter: Pathology and genetics of tumours of the breast and female genital organs. Lyon, IAPS Press, 2003 2003.
27. Heintz AP, Odicino F, Maisonneuve P, Beller U, Benedet JL, Creasman WT, Ngan HY, Sideri M and Pecorelli S: Carcinoma of the ovary. J Epidemiol Biostat 6: 107-138, 2001.

28. Miglarese MR, Mannion-Henderson J, Wu H, Parsons JT and Bender TP: The protein tyrosine kinase substrate cortactin is differentially expressed in murine B lymphoid tumors. Oncogene 9: 1989-1997, 1994.

29. Zhan X, Haudenschild CC, Ni Y, Smith E and Huang C: Upregulation of cortactin expression during the maturation of megakaryocytes. Blood 89: 457-464, 1997.

30. Mizutani K, Miki H, He H, Maruta $\mathrm{H}$ and Takenawa $\mathrm{T}$ : Essential role of neural Wiskott-Aldrich syndrome protein in podosome formation and degradation of extracellular matrix in src-transformed fibroblasts. Cancer Res 62: 669-674, 2002.

31. Schuuring E, Verhoeven E, Litvinov S and Michalides RJ: The product of the EMS1 gene, amplified and overexpressed in human carcinomas, is homologous to a v-src substrate and is located in cell-substratum contact sites. Mol Cell Biol 13: 2891-2898, 1993.

32. Gattazzo C, Martini V, Frezzato F, Trimarco V, Tibaldi E, Castelli M, Facco M, Zonta F, Brunati AM, Zambello R, et al: Cortactin, another player in the Lyn signaling pathway, is over-expressed and alternatively spliced in leukemic cells from patients with B-cell chronic lymphocytic leukemia. Haematologica 99: 1069-1077, 2014

33. Xu XZ, Garcia MV, Li TY, Khor LY, Gajapathy RS, Spittle C, Weed S, Lessin SR and Wu H: Cytoskeleton alterations in melanoma: Aberrant expression of cortactin, an actin-binding adapter protein, correlates with melanocytic tumor progression. Mod Pathol 23: 187-196, 2010.

34. Soslow RA: Histologic subtypes of ovarian carcinoma: An overview. Int J Gynecol Pathol 27: 161-174, 2008.

35. Taniuchi I, Kitamura D, Maekawa Y, Fukuda T, Kishi H and Watanabe T: Antigen-receptor induced clonal expansion and deletion of lymphocytes are impaired in mice lacking HS1 protein, a substrate of the antigen-receptor-coupled tyrosine kinases. EMBO J 14: 3664-3678, 1995.

36. Park SH, Song JY, Kim YK, Heo JH, Kang H, Kim G, An HJ and Kim TH: Fascin1 expression in high-grade serous ovarian carcinoma is a prognostic marker and knockdown of fascin1 suppresses the proliferation of ovarian cancer cells. Int $\mathrm{J}$ Oncol 44: 637-646, 2014.

37. Hashimoto Y, Skacel $M$ and Adams JC: Roles of fascin in human carcinoma motility and signaling: Prospects for a novel biomarker? Int J Biochem Cell Biol 37: 1787-1804, 2005.

38. Rottner K and Stradal TE: Actin dynamics and turnover in cell motility. Curr Opin Cell Biol 23: 569-578, 2011. 